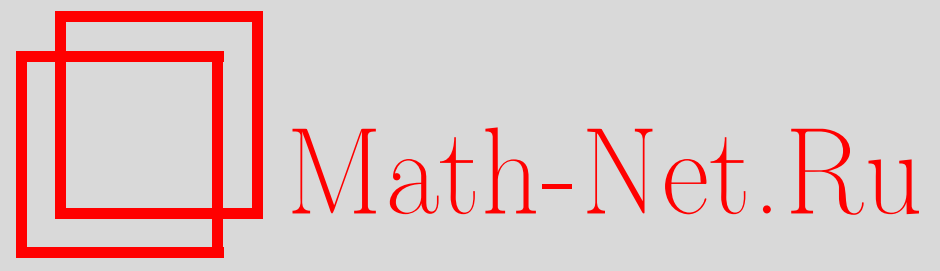

Д. Н. Бабин, О разрешимости проблемы полноты для специальных систем автоматных функций, Дискрет. матем., 1996, том 8, выпуск 4, 79-91

DOI: https://doi.org/10.4213/dm542

Использование Общероссийского математического портала Math-Net.Ru подразумевает, что вы прочитали и согласны с пользовательским соглашением http://www.mathnet.ru/rus/agreement

Параметры загрузки:

IP : 35.173 .219 .149

26 апреля 2023 г., $14: 21: 56$

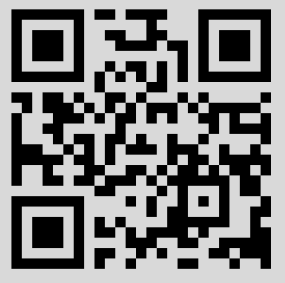




\title{
О разрешимости проблемы полноты для специальных систем автоматных функций
}

\author{
(C) 1996 г. Д. Н. Бабин
}

\begin{abstract}
Рассматриваются системы автоматных функций вида $M=\Phi \cup \nu$, где $\Phi$ - некоторый класс Поста, а $\nu$ - конечная система автоматных функций. Показано, что если $\Phi=M, D, C, F^{2}$, то проблема полноты и $A$-полноты для системы $M$ алгоритмически разрешима.

Работа выполнена при поддержке Российского фонда фундаментальных исследований, проект 95-01-01102.
\end{abstract}

\section{1. Введение}

Известно, что решение задачи о полноте относительно операций суперпозиции и обратной связи для систем автоматных функций наталкивается на существенные трудности. Так, в [1] установлена континуальность всякой критериальной системы для этой задачи, а позднее в [2] установлена ее алгоритмическая неразрешимость для конечных систем автоматных функций. Вместе с тем, для систем автоматов, содержащих все булевы функции, указанная задача алгоритмически разрешима [3]. Естественно исследовать на полноту системы вида $M=\Phi \cup \nu$, где $\Phi$ - некоторый класс Поста, а $\nu$ - конечная система автоматных функций.

Проблема $A$-полноты для систем автоматных функций алгоритмически неразрешима в общей постановке [4] и разрешима для систем, содержащих все булевы функции [5]. В [10] было показано, что для классов Поста $\Phi$ типа $F^{\infty}, S$, $P$ и $O$ проблема полноты и проблема $A$-полноты алгоритмически неразрешима. Из результата настоящей работы следует, что для

$$
\Phi \in\left\{M_{1}, M_{2}, M_{3}, M_{4}, D_{1}, D_{2}, D_{3}, C_{1}, C_{2}, C_{3}, C_{4}, F_{i}{ }^{2}, i=1,2, \ldots, 8\right\}
$$

проблема полноты и $A$-полноты алгоритмически разрешима.

\section{2. Основные понятия и результаты}

Мы будем использовать обозначения из работ [6, 7]. Пусть $E_{2}=\{0,1\}, E_{2}^{*}-$ множество всех слов $a(1) a(2) \ldots a(s)$, где $a(j) \in E_{2}, j=1,2, \ldots s, E_{2}^{\tau}-$ множество всех слов $a(1) \ldots a(\tau)$ длины $\tau$. Пусть $f:\left(E_{2}^{*}\right)^{n} \rightarrow\left(E_{2}^{*}\right)^{m}$ - автоматная функция 
(а-функция), т.е. она задается рекуррентно соотношениями

$$
\begin{aligned}
q(1) & =q_{1} \\
q(t+1) & =\varphi\left(q(t), a_{1}(t), \ldots, a_{n}(t)\right), \\
b_{j}(t) & =\psi_{j}\left(q(t), a_{1}(t), \ldots, a_{n}(t)\right), \quad j=1, \ldots, m,
\end{aligned}
$$

где $q \in Q=\left\{q_{1}, \ldots, q_{r}\right\}$. Параметр $q$ называется состоянием $a$-функции $f, q_{1}$ ее начальным состоянием, вектор-буквы $a=\left(a_{1}, \ldots, a_{n}\right)$ и $b=\left(b_{1}, \ldots, b_{m}\right)$ называются входной и выходной буквами, а сверхслова $a(1) a(2) \ldots$ и $b(1) b(2) \ldots$ входным и выходным сверхсловами, соответственно. Класс всех $a$-функций обозначим через $P$. В этом классе обычным образом введем операции суперпозиции и обратной связи. Для суперпозиции будем использовать модификации операций из [8]:

$$
\begin{aligned}
(\gamma f)\left(x_{1}, \ldots, x_{n}\right) & =f\left(x_{2}, x_{3}, \ldots, x_{n}, x_{1}\right), \\
(\epsilon f)\left(x_{1}, \ldots, x_{n}\right) & =f\left(x_{2}, x_{1}, x_{3}, \ldots, x_{n}\right), \\
(\Omega f)\left(x_{1}, \ldots, x_{n-1}\right. & =f\left(x_{1}, x_{1}, x_{2}, \ldots, x_{n-1}\right), \\
(\nabla f)\left(x_{1}, x_{2}, \ldots, x_{n+1}\right) & =f\left(x_{2}, x_{3}, \ldots, x_{n+1}\right) \\
(f * g)\left(x_{1}, x_{2}, \ldots, x_{m+n-1}\right) & =f\left(g\left(x_{1}, \ldots, x_{m}\right), x_{m+1}, \ldots, x_{m+n-1}\right) .
\end{aligned}
$$

Операция обратной связи (о.с.), примененная к $i$-й входной и $j$-й выходной переменным $а$-функции $f\left(x_{1}, \ldots, x_{n}\right)=\left(y_{1}, \ldots, y_{m}\right)$, задает $a$-функцию $g\left(x_{1}, \ldots, x_{i-1}, x_{j+1}, \ldots, x_{n}\right)=\left(y_{1}, \ldots, y_{j-1}, y_{j+1}, \ldots, y_{m}\right)$, вычисляемую алгоритмически следующим образом. Считаем, что о.с. применима к $f$ в состоянии $q$, если $\psi_{j}$ в уравнении (1) фиктивно зависит от $a_{i}$ при $q(t)=q$, а вычисление $b_{s}(t)$ осуществляется по схеме

$$
\begin{aligned}
q(1)= & q_{1} \\
q(t+1)= & \varphi\left(q(t), a_{1}(t), \ldots, a_{i-1}(t), \psi_{j}\left(q(t), a_{1}(t), \ldots a_{i-1}(t),\right.\right. \\
& \left.\left.\quad a_{i+1}(t), \ldots, a_{n}(t)\right), a_{i+1}(t), \ldots, a_{n}(t)\right), \\
b_{s}(t)= & \psi_{s}\left(q(t), a_{1}(t), \ldots, a_{i-1}(t), \psi_{j}\left(q(t), a_{1}(t), \ldots, a_{i-1}(t),\right.\right. \\
& \left.\left.a_{i+1}(t), \ldots, a_{n}(t)\right), a_{i+1}(t), \ldots, a_{n}(t)\right), \quad s=1,2, \ldots, j-1, j+1, \ldots, m .
\end{aligned}
$$

Считаем, что о.с. применима к $f$, если она применима в начальном состоянии $q_{1}$, и из ее применимости в состоянии $q(t)$ следует применимость в состоянии $q(t+1)$. Пусть $M \subseteq P$, обозначим через $[M]$ множество всех $a$-функций, получающихся из $M$ с помощью операций суперпозиции и обратной связи. Множество $M$ называется полным, если $[M]=P$. Проблема полноты для $P$ состоит в описании всех полных множеств $M$.

Пусть $\tau$ - натуральное число, $f\left(x_{1}, \ldots, x_{n}\right)$ - некоторая автоматная функция, $f^{\tau}:\left(E_{2}{ }^{\tau}\right)^{n} \rightarrow\left(E_{2}{ }^{\tau}\right)^{m}$ - ограничение этой функции на множество слов длины $\tau$. Скажем, что а-функции $f\left(x_{1}, \ldots, x_{n}\right)$ и $g\left(x_{1}, \ldots, x_{n}\right)$ tau-равны, если $f^{\tau}=g^{\tau}$. Обозначим через $[M]_{\tau}$ множество всех $a$-функций, $\tau$-равных получающимся из $M$ с помощью операций суперпозиции и обратной связи. Известно [7 ], что результат применения о.с. $\tau$-равен $\tau$ применениям суперпозиции. Множество $M$ называется $\tau$-полным, если $[M]_{\tau}=P$. Множество $M$ называется $A$-полным, если $[M]_{\tau}=P$ при всех $\tau$. Положим $[M]_{A}=\bigcap[M]_{\tau}$. Проблема $A$-полноты для $P$ состоит в описании всех $A$-полных множеств $M$. Очевидно, что полное множество 
$M$ является $A$-полным. Обозначим через $P^{(i)}$ класс всех $a$-функций с не более, чем $i$ состояниями.

Известно, что $\left[P^{(2)}\right]=P,\left[P^{(1)}\right]=P^{(1)}$. Истинностными называются $a$ функции из $P^{(1)}$. Как отмечалось, для систем $a$-функций вида $P^{(1)} \cup \nu$, где $\nu$ - конечное множество, проблема полноты [3] и $A$-полноты [5] алгоритмически разрешимы. Пусть $\Phi$ - замкнутый класс истинностных функций из $P^{(1)}$. Рассмотрим проблемы полноты и $A$-полноты для систем вида $\Phi \cup \nu$, которые мы будем называть проблемами $\Phi$-полноты и $\Phi$ - $A$-полноты, соответственно. В данной работе рассматривается случай, когда $\Phi$ является классом Поста типа $M$, $D, C, F^{2}$. Классы типа $M$ суть $M_{1}, M_{2}, M_{3}, M_{4}$, типа $C$ суть $C_{1}, C_{2}, C_{3}, C_{4}$, типа $D$ суть $D_{1}, D_{2}, D_{3}$, типа $F^{2}$ суть $F_{i}^{2}, i=1,2, \ldots, 8[6]$, где

$$
\begin{array}{rlrl}
M_{1} & =[\{x y, x \vee y, 0,1\}], & M_{2} & =[\{x y, x \vee y, 1\}], \\
M_{3} & =[\{x y, x \vee y, 0\}], & M_{4} & =[\{x y, x \vee y\}], \\
C_{1} & =P^{(1)}, & C_{2} & =[\{x y, x+y+1\}], \\
C_{3} & =[\{x y, x+y\}], & C_{4} & =[\{x \vee y, x(y+z+1)\}], \\
D_{1} & =[\{x y \vee x \bar{z} \vee y \bar{z}\}], & D_{2} & =[\{x y \vee x z \vee y z\}], \\
D_{3} & =[\{x \bar{y} \vee x \bar{z} \vee \bar{y} \bar{z}\}], & & \\
F_{1}^{2} & =[\{x \vee y \bar{z}, h(x, y, z)\}], & F_{2}^{2}=[\{x \vee y z, h(x, y, z)\}], \\
F_{3}^{2} & =[\{1, h(x, y, z)\}], & F_{4}^{2} & =[\{x \vee \bar{y}, h(x, y, z)\}] ;
\end{array}
$$

Классы $F_{5}^{2}, F_{6}^{2}, F_{7}^{2}, F_{8}^{2}$ двойственны к классам $F_{1}^{2}, F_{2}^{2}, F_{3}^{2}, F_{4}^{2}$, соответственно, $h(x, y, z)=x y \vee x z \vee y z$. Справедливы следующие теоремы.

Теорема 1. Проблема Ф-полноты для каждого класса $\Phi$ muпа $M, C, D, F^{2}$ aлгоритмически разрешима.

Теорема 2. Проблема Ф-А-полноть для каждого класса $\Phi$ muпа $M, C, D, F^{2}$ алгоритмически разрешима.

Заметим, что если проблема $\Phi$-полноты ( $\Phi$ - $A$-полноты) алгоритмически разрешима для $\Phi=F_{1}$, то она разрешима для всякого замкнутого класса $F_{2} \supseteq F_{1}$ и двойственного к $F_{1}$ замкнутого класса $F_{1}^{*}$. Отсюда следует, что для доказательства теорем достаточно установить их справедливость для $\Phi=D_{2}$.

\section{3. Основные леммы}

Через $\mathrm{N}$ обозначим множество натуральных чисел. Положим

$$
h\left(x_{1}, x_{2}, x_{3}\right)=x_{1} x_{2} \vee x_{1} x_{3} \vee x_{2} x_{3},
$$

и через $\bar{X}, \overline{1}, 0, H$ обозначим автоматные интерпретации соответствующих истинностных функций $\bar{x}, 1,0, h$. Для натуральных $D, N$ автоматную функцию с уравнениями (1), где

$$
m=n=D+N, \quad Q=\{1, \ldots, D+N\}
$$


и для любого $a \in E_{2}^{D+N}$

$$
\begin{aligned}
\varphi(i, a) & =i+1, \quad i<D+N, \\
\varphi(D+N, a) & =D,
\end{aligned}
$$

$\psi(i, a)=0 \ldots 010 \ldots 0$, где 1 стоит на $i$-м месте, назовем $(D, N)$-счетчиком и обозначим через $B_{D, N}$. Если же при этом $\psi\left(i,\left(a_{1}, \ldots a_{D+N}\right)\right)=a_{i}$, где $a_{1}, \ldots, a_{D+N} \in$ $E_{2}$, то назовем ее $(D, N)$-селектором и обозначим через $C_{D, N}$.

Без ограничения общности, будем исследовать на полноту и $A$-полноту системы вида $\{H, f\}$ состоящие из двух $a$-функций. Пусть $j$ и $s-$ натуральные числа, $j \leqslant s$. Последовательность

$$
(a, b),(a(1), b(1)), \ldots,(a(s), b(s)),
$$

где $a, a(1), \ldots, a(s) \in E_{2}^{n}, q(1), \ldots q(s) \in Q, b, b(1), \ldots, b(s) \in E_{2}^{m}, b(i)=\psi(q(i), a(i))$, $i=1, \ldots, s, q(i+1)=\varphi(q(i), a(i)), i=1, \ldots, s-1, b=\psi(q(j), a)), q(1)=q_{1}$, назовем $(s, j)$-әкспериментом с $a$-функцией $f$. Если, кроме того, $\varphi(q(s), a(s))=q(D), 1 \leqslant$ $D \leqslant s$, то назовем $(2)(D, s, j)$-экспериментом с $a$-функцией $f$.

Будем использовать знаки $\wedge$ для обозначения покомпонентной конъюнкции, a $\vee$ для дизъюнкции произвольного числа векторов из $E_{2}^{k}$ при произвольном $k$. Для обозначения наборов $11 \ldots 1$ и $00 \ldots 0$ произвольной длины, там где әто не приводит к недоразумению, будем использовать, соответственно, $\mathbf{1}$ и $\mathbf{0}$. Для $a \in E_{2}^{k}$ и $\delta \in E_{2}$ определим $a^{\delta}$ и $a \delta \in E_{2}^{k}$, полагая

$$
a^{\delta}=\left\{\begin{array}{ll}
1 & \text { при } \delta=0, \\
a & \text { при } \delta=1,
\end{array} \quad a \delta= \begin{cases}0 & \text { при } \delta=0, \\
a & \text { при } \delta=1 .\end{cases}\right.
$$

Пусть $\xi, \eta \in E_{2}^{s}$. Скажем, что $a$-функция $f$ сохраняет на $(D, s, j)$-әксперимен$\operatorname{tax}((s, j)$-экспериментах) прямую зависимость с коэффициентами $(\xi, \eta)$, если для любого эксперимента (2) с $a$-функцией $f$

$$
\begin{aligned}
\left(\left(a \vee \bigwedge_{1}^{s} a(i)^{\xi(i)}\right)=1\right) & \wedge\left(\bar{a}\left(\bigvee_{1}^{s} a(i) \eta(i)\right)=0\right) \\
& \Rightarrow\left(\left(b \vee \bigwedge_{1}^{s} b(i)^{\xi(i)}\right)=1\right) \wedge\left(\bar{b}\left(\bigvee_{1}^{s} b(i) \eta(i)\right)=0\right)
\end{aligned}
$$

Скажем, что $a$-функция $f$ сохраняет на $(D, s, j)$-әкспериментах $((s, j)$-әкспериментах) двойственную зависимость с коэффициентами $(\xi, \eta)$, если для любого эксперимента (2) с $a$-функцией $f$

$$
\begin{aligned}
\left(a\left(\bigvee_{1}^{s} a(i) \xi(i)\right)=0\right) & \wedge\left(\left(\bar{a} \vee \bigwedge_{1}^{s} a(i)^{\eta(i)}\right)=1\right) \\
& \Rightarrow\left(b\left(\bigvee_{1}^{s} b(i) \xi(i)\right)=0\right) \wedge\left(\left(\bar{b} \vee \bigwedge_{1}^{s} b(i)^{\eta(i)}\right)=1\right)
\end{aligned}
$$

Скажем, что $a$-функция $f$ сохраняет 1 на әкспериментах (2), если $a=1 \Rightarrow b=1$; сохраняет $\mathbf{0}$, если $a=0 \Rightarrow b=0$. 
Пусть $1 \leqslant i, j \leqslant s$; скажем, что $a$-функция $f$ сохраняет на $(D, s, j)$ экспериментах $((s, j)$-әкспериментах) тождественную зависимость в $i$-й момент, если для любого указанного әксперимента $(2) a=a(i) \Rightarrow b=b(i)$, сохраняет инверсную зависимость в $i$-й момент, если для любого эксперимента (2) $a=\bar{a}(i) \Rightarrow b=\bar{b}(i)$.

Предложение 1. Для любых фиксированных натуральных $D, s, j, i, 1 \leqslant i, j \leqslant s$, $D \leqslant s, \xi, \eta \in E_{2}^{s}$ для әкспериментов (2) сөойства а-функций сохранять прямую зависимость с коэффициентами $(\xi, \eta)$, дөойственную зависимость с коэффициентами $(\xi, \eta)$, тохсестөенную зависимость в $i$-й момент инверсную зависимость в $i$-й момент не нарушаются при операциях суперпозиции и обратной связи. $A$ функция $H$ такжсе сохраняет на өсех әкспериментах (2) указанные зависимости.

Пусть $Q_{t} \subseteq Q$ - множество состояний $a$-функции $f$, достижимых из начального состояния $q_{1}$ за $t$ тактов. Для $p_{1}, p_{2} \in Q$ и натурального $s$ обозначим через $L\left(p_{1}, p_{2}, s\right)$ множество слов $\beta \in\left(E_{2}^{m+n}\right)^{s}$ вида

$$
\beta=\beta(1) \beta(2) \ldots \beta(s), \quad \beta(i)=(a(i), b(i)), \quad a(i) \in E_{2}^{n}, \quad b(i) \in E_{2}^{m}, \quad i=1,2 \ldots s,
$$

при условии, что

$$
\varphi\left(p_{1}, a(1), \ldots, a(s)\right)=p_{2}, \quad b(1), b(2), \ldots, b(s)=\bar{\psi}\left(p_{1}, a(1), a(2), \ldots, a(s)\right) .
$$

Пусть $\xi \in E_{2}^{s}, \beta(1), \ldots \beta(s) \in\left(E_{2}^{m+n}\right)^{s}$; положим $\mu(\beta, \xi)=\beta(l)$, где $l$ - позиция первой в слове $\xi$ единицы, $\mu(\beta, 0 \ldots 0)=\Lambda$, где $\Lambda$ - пустая буква. Для $\xi, \eta \in E_{2}^{s}$ положим

$$
\begin{aligned}
X\left(p_{1}, p_{2}, \xi, \eta\right) & =\left\{\left(\bigwedge_{1}^{s} \beta(i)^{\xi(i)}, \bigvee_{1}^{s} \beta(i) \eta(i)\right) \mid \beta(1) \ldots \beta(s) \in L\left(p_{1}, p_{2}, s\right)\right\} \\
Y\left(p_{1}, p_{2}, \xi, \eta\right) & =\left\{\left(\bigvee_{1}^{s} \beta(i) \xi(i), \bigwedge_{1}^{s} \beta(i)^{\eta(i)}\right) \mid \beta(1) \ldots \beta(s) \in L\left(p_{1}, p_{2}, s\right)\right\} \\
Z\left(p_{1}, p_{2}, \xi\right) & =\left\{\mu(\beta, s) \mid \beta(1) \ldots \beta(s) \in L\left(p_{1}, p_{2}, s\right)\right\}
\end{aligned}
$$

Определим полные графы $G X(\xi, \eta), G Y(\xi, \eta)$ и $G Z(\xi)$ с метками на ребрах и множеством вершин $Q$. В графе $G X(\xi, \eta)$ ребро $\left(p_{1}, p_{2}\right)$ имеет метку $X\left(p_{1}, p_{2}, \xi, \eta\right)$, в графе $G Y(\xi, \eta)$ метка равна $Y\left(p_{1}, p_{2}, \xi, \eta\right)$, а в графе $G Z(\xi)$ она равна $Z\left(p_{1}, p_{2}, \xi\right)$. Пусть

$$
\begin{aligned}
& \widehat{G X}=\left\{G X(\xi, \eta) \mid(\xi, \eta) \in\left(E_{2}^{2}\right)^{*}\right\} \\
& \widehat{G Y}=\left\{G Y(\xi, \eta) \mid(\xi, \eta) \in\left(E_{2}^{2}\right)^{*}\right\}, \\
& \widehat{G Z}=\left\{G Z(\xi) \mid \xi \in E_{2}^{*}\right\} .
\end{aligned}
$$

Определим автоматы без выхода

$$
\begin{aligned}
& \widehat{A X}=\left(E_{2}^{2}, \widehat{G X}, \phi_{X}, G_{0}\right), \\
& \widehat{A Y}=\left(E_{2}^{2}, \widehat{G Y}, \phi_{Y}, G_{0}\right), \\
& \widehat{A Z}=\left(E_{2}, \widehat{G Z}, \phi_{Z}, G_{0}\right),
\end{aligned}
$$


где

$$
\begin{aligned}
\phi_{X}(G X(\xi, \eta),(x, y)) & =G X(\xi x, \eta y), \\
\phi_{Y}(G Y(\xi, \eta),(x, y)) & =G Y(\xi x, \eta y), \\
\phi_{Z}(G Z(\xi), x) & =G Z(\xi x), \quad x, y \in E_{2},
\end{aligned}
$$

пусть $G_{0}$ - полный граф с пустыми метками. Функции $\phi_{X}, \phi_{Y}$ и $\phi_{Z}$ определены корректно. Обозначим через $G X_{t}, G Y_{t}$ и $G Z_{t}$ множества состояний автоматов $\widehat{A X}, \widehat{A Y}$ и $\widehat{A Z}$, соответственно, достижимых из начального состояния за $t$ тактов. Последовательности $G_{0}, G X_{1}, G X_{2}, \ldots, G_{0}, G Y_{1}, G Y_{2}, \ldots$ и $G_{0}, G Z_{1}, G Z_{2}, \ldots$ периодичны и, без ограничения общности, имеют период и предпериод $\varrho$.

Пусть $\tilde{\jmath}, \tilde{D}, \tilde{s} \in \mathbf{N}, \tilde{j}<\tilde{D}$, и а-функция $f$ на $(\tilde{D}, \tilde{s}, \tilde{\jmath})$-экспериментах сохраняет прямую зависимость с коэффициентами $\left(\tilde{\xi}_{1} \tilde{\xi}_{2} \tilde{\xi}_{3}, \tilde{\eta}_{1} \tilde{\eta}_{2} \tilde{\eta}_{3}\right),\left|\left(\tilde{\xi}_{1}, \tilde{\eta}_{1}\right)\right|=\tilde{\jmath},\left|\left(\tilde{\xi}_{2}, \tilde{\eta}_{2}\right)\right|=$ $\tilde{D}-\tilde{j},\left|\left(\tilde{\xi}_{3}, \tilde{\eta}_{3}\right)\right|=\tilde{s}-\tilde{D}$.

Пусть коэффициенты $\left(\xi_{1} \xi_{2} \xi_{3}, \eta_{1} \eta_{2} \eta_{3}\right)$ таковы, что

$$
\begin{gathered}
\phi_{X}\left(G_{0},\left(\xi_{1}, \eta_{1}\right)\right)=\phi_{X}\left(G_{0},\left(\tilde{\xi}_{1}, \tilde{\eta}_{1}\right)\right)=G_{1}, \\
\phi_{X}\left(G_{0},\left(\xi_{2}, \eta_{2}\right)\right)=\phi_{X}\left(G_{0},\left(\tilde{\xi}_{2}, \tilde{\eta}_{2}\right)\right)=G_{2}, \\
\phi_{X}\left(G_{0},\left(\xi_{3}, \eta_{3}\right)\right)=\phi_{X}\left(G_{0},\left(\tilde{\xi}_{3}, \tilde{\eta}_{3}\right)\right)=G_{3}, \\
\left|\left(\xi_{1}, \eta_{1}\right)\right|=j, \quad\left|\left(\xi_{2}, \eta_{2}\right)\right|=D-j, \quad\left|\left(\xi_{3}, \eta_{3}\right)\right|=s-\dot{D} ;
\end{gathered}
$$

тогда $a$-функция $f$ по построению автомата $\widehat{A X}$ сохраняет прямую зависимость с коэффициентами $\left(\xi_{1} \xi_{2} \xi_{3}, \eta_{1} \eta_{2} \eta_{3}\right)$ на $(D, s, j)$-экспериментах. Сказанное справедливо как для случая $\tilde{\jmath} \geqslant \tilde{D}$, так и для двойственной зависимости.

Пусть $L X-$ множество троек $\left(G_{1}, G_{2}, G_{3}\right)$ состояний автомата $\widehat{A X}(\widehat{A Y})$, для любых $\left(\xi_{1}, \eta_{1}\right),\left(\xi_{2}, \eta_{2}\right),\left(\xi_{3}, \eta_{3}\right)$ обладающих свойством

$$
\phi_{X}\left(G_{0},\left(\xi_{1}, \eta_{1}\right)\right)=G_{1}, \quad \phi_{X}\left(G_{0},\left(\xi_{2}, \eta_{2}\right)\right)=G_{2}, \quad \phi_{X}\left(G_{0},\left(\xi_{3}, \eta_{3}\right)\right)=G_{3} ;
$$

$a$-функция $f$ сохраняет прямую (двойственную) зависимость с коэффициентами $\left(\xi_{1} \xi_{2} \xi_{3}, \eta_{1} \eta_{2} \eta_{3}\right)$ на $\left(\left|\xi_{1} \xi_{2}\right|,\left|\xi_{1} \xi_{2} \xi_{3}\right|,\left|\xi_{1}\right|\right)$-экспериментах, $R X(R Y)$ - аналогичное множества троек состояний автомата $\widehat{A X}(\widehat{A Y})$ для сохранения прямой (двойственной) зависимости с коэффициентами $\left(\xi_{1} \xi_{2} \xi_{3}, \eta_{1} \eta_{2} \eta_{3}\right)$ на $\left(\left|\xi_{1}\right|,\left|\xi_{1} \xi_{2} \xi_{3}\right|,\left|\xi_{1} \xi_{2}\right|\right)$ экспериментах.

Пусть $T L(I L)$ - множество троек $G_{1}, G_{2}, G_{3}$ состояний автомата $\widehat{A Z}$ таких, что для $\xi_{1}, \xi_{2}, \xi_{3}$ :

$$
\phi_{Z}\left(G_{0}, \xi_{l}\right)=G_{l}, \quad l=1,2,3, \quad \xi_{1} \xi_{2} \xi_{3}=0 \ldots 010 \ldots 0
$$

на всех $\left(\left|\xi_{1} \xi_{2}\right|,\left|\xi_{1} \xi_{2} \xi_{3}\right|,\left|\xi_{1}\right|\right)$-экспериментах сохраняется тождественная (инверсная) зависимость в $i$-й момент, где $i$ - позиция первой единицы в слове $\xi_{1} \xi_{2} \xi_{3}$. $T R, I R$ - аналогичные множества для $\left(\left|\xi_{1}\right|,\left|\xi_{1} \xi_{2} \xi_{3}\right|,\left|\xi_{1} \xi_{2}\right|\right)$-экспериментов.

Пусть $D, k, N \in \mathbf{N}$; положим

$$
\begin{aligned}
& \Omega_{1}(N, D, k)=\bigcup_{j=1}^{D-1}\left\{\left(G_{1}, G_{2}, G_{3}\right) \in L X \mid G_{1} \in G X_{j}, G_{2} \in G X_{D-j}, G_{3} \in G X_{k N}\right\}, \\
& \Omega_{2}(N, D, k)=\bigcup_{j=1}^{D-1}\left\{\left(G_{1}, G_{2}, G_{3}\right) \in L Y \mid G_{1} \in G Y_{j}, G_{2} \in G Y_{D-j}, G_{3} \in G Y_{k N}\right\},
\end{aligned}
$$




$$
\begin{aligned}
\Omega_{3}(N, D, k)= & \bigcup_{j=D}^{D+k N}\left\{\left(G_{1}, G_{2}, G_{3}\right) \in R X \mid G_{1} \in G X_{D}, G_{2} \in G X_{j-D}, G_{3} \in G X_{k N-j+D}\right\}, \\
\Omega_{4}(N, D, k)= & \bigcup_{j=D}^{D+k N}\left\{\left(G_{1}, G_{2}, G_{3}\right) \in R Y \mid G_{1} \in G Y_{D}, G_{2} \in G Y_{j-D}, G_{3} \in G Y_{k N-j+D}\right\}, \\
\Omega_{5}(N, D, k)= & \bigcup_{j=1}^{D-1}\left\{\left(G_{1}, G_{2}, G_{3}\right) \in T L \mid G_{1} \in G Z_{j}, G_{2} \in G Z_{D-j}, G_{3} \in G Z_{k N}\right\}, \\
\Omega_{6}(N, D, k)= & \bigcup_{j=D}^{D+k N}\left\{\left(G_{1}, G_{2}, G_{3}\right) \in T R \mid G_{1} \in G Z_{D}, G_{2} \in G Z_{j-D}, G_{3} \in G Z_{k N-j+D}\right\}, \\
\Omega_{7}(N, D, k)= & \bigcup_{j=1}^{D-1}\left\{\left(G_{1}, G_{2}, G_{3}\right) \in I L \mid G_{1} \in G Z_{j}, G_{2} \in G Z_{D-j}, G_{3} \in G Z_{k N}\right\}, \\
\Omega_{8}(N, D, k)= & \bigcup_{j=D}^{D+k N}\left\{\left(G_{1}, G_{2}, G_{3}\right) \in I R \mid G_{1} \in G Z_{D}, G_{2} \in G Z_{j-D}, G G_{3} \in G Z_{k N-j+D}\right\} .
\end{aligned}
$$

Пусть $R$ - число, кратное длинам всех простых циклов в диаграмме автомата $\widehat{A Z}$, большее, чем число $|\widehat{G X}|$.

Предложение 2. Функция $\Omega_{l}(N, D, k), l=1,2, \ldots 8$, периодична с периодом $R \varrho$ по кажсдому из своих аргументов.

Лемма 1. Система $\{H, f\}$ полна тогда и только тогда, когда для любого натурального $N$ существуют такие $D$ u $k$, что $\Omega_{l}(N, D, k)=\varnothing, l=1, \ldots 8, u$ для любого $j \leqslant D+k N f$ не сохраняет 1 и не сохраняет 0 на $(D, s, j)$-экспериментах.

Проведем аналогичное построение для $A$-полноты. Пусть $A L X(A L Y)-$ множество пар $\left(G_{1}, G_{2}\right)$ состояний автомата $\widehat{A X}(\widehat{A Y})$ таких,что для любых $\left(\xi_{1}, \eta_{1}\right)$, $\left(\xi_{2}, \eta_{2}\right)$, обладающих свойством

$$
\phi_{X}\left(G_{0},\left(\xi_{1}, \eta_{1}\right)\right)=G_{1}, \quad \phi_{X}\left(G_{0},\left(\xi_{2}, \eta_{2}\right)\right)=G_{2}
$$

$a$-функция $f$ сохраняет прямую (двойственную) зависимость с коэффициентами $\left(\xi_{1} \xi_{2}, \eta_{1} \eta_{2}\right)$ на $\left(\left|\xi_{1} \xi_{2}\right|,\left|\xi_{1}\right|\right)$-экспериментах. Пусть $A T(A I)-$ множество пар $\left(G_{1}, G_{2}\right)$, состояний $\widehat{A Z}$ таких, что для

$$
\xi_{1} \xi_{2}=0 \ldots 010 \ldots 0, \quad \phi_{Z}\left(G_{0}, \xi_{1}\right)=G_{1}, \quad \phi_{Z}\left(G_{0}, \xi_{2}\right)=G_{2}
$$

на всех $\left(\left|\xi_{1} \xi_{2}\right|,\left|\xi_{1}\right|\right)$-экспериментах сохраняется тождественная (инверсная) зависимость в $i$-й момент, где $i$ - позиция единственной в слове $\xi_{1} \xi_{2}$ единицы. Положим

$$
\begin{aligned}
& \omega_{1}(s)=\bigcup_{j=1}^{s}\left\{\left(G_{1}, G_{2}\right) \in A L X \mid G_{1} \in G X_{j}, G_{2} \in G X_{s-j}\right\} \\
& \omega_{2}(s)=\bigcup_{j=1}^{s}\left\{\left(G_{1}, G_{2}\right) \in A L Y \mid G_{1} \in G Y_{j}, G_{2} \in G Y_{s-j}\right\}
\end{aligned}
$$




$$
\begin{aligned}
& \omega_{3}(s)=\bigcup_{j=1}^{s}\left\{\left(G_{1}, G_{2}\right) \in A T \mid G_{1} \in G Z_{j}, G_{2} \in G Z_{s-j}\right\} \\
& \omega_{4}(s)=\bigcup_{j=1}^{s}\left\{\left(G_{1}, G_{2}\right) \in A I \mid G_{1} \in G Z_{j}, G_{2} \in G Z_{s-j}\right\} .
\end{aligned}
$$

Предложение 3. Для любого натурального $s$

$$
\omega_{l}(s+R \varrho)=\omega_{l}(s), \quad l=1,2,3,4
$$

Лемма 2. Система $\{H, f\}$ A-полна тогда и только тогда, когда $\omega_{l}(s)=\varnothing, l=$ $1,2,3,4$, для любого $s, u f$ не сохраняет 1 и не сохраняет 0 на $(s, j)$-әкспериментах для любого $j \leqslant s$.

Из лемм 1, 2 и утверждений 1,2 и 3 следует доказательство теорем 1 и 2.

\section{4. Доказательства лемм}

Доказательство предлохсения 1. Пусть $f_{1}=f_{2}\left(f_{3}\right)$ и для $f_{2}$ и $f_{3}$ выполнено (3), тогда для всякого $(D, s, j)$-эксперимента с $f_{1}$ также будет выполнено (3).

Пусть $a$-функция $f$ получилась операцией обратной связи из $a$-функции $g$ и при этом эксперимент (2) получился из эксперимента (4)

$$
((a, e),(b, e))((a(1), e(1)),(b(1), e(1))), \ldots((a(s), e(s)),(b(s), e(s))) .
$$

Пусть для (2) справедливо равенство

$$
a\left(\bigvee_{1}^{s} a(i) \xi(i)\right)=0, \quad \bar{a} \vee \bigwedge_{1}^{s} a(i)^{\eta(i)}=1
$$

Рассмотрим эксперимент

$$
((a, 1),(\widetilde{b}, \widetilde{e}))((a(1), e(1)),(b(1), e(1))), \ldots((a(s), e(s)),(b(s), e(s))) .
$$

Для него выполнено соотношение (3), значит

$$
\tilde{b}\left(\bigvee_{1}^{s} b(i) \xi(i)\right)=0, \quad \overline{\tilde{b}} \vee \bigwedge_{1}^{s} b(i)^{\eta(i)}=1
$$

По определению операции обратной связи $\tilde{e}=e$, значит и $\tilde{b}=b$, то есть $a$ функция $f$ сохраняет (3) на $(D, s, j)$-экспериментах. Случаи сохранения двойственной, тождественной и инверсной зависимости доказываются аналогично. Предложение доказано. 
Доказательство леммы 1. Докажем необходимость. Пусть через $H$ и $f$ выражена $a$-функция $g$, реализующая $\bar{X}$, тогда существуют такие натуральные $D_{1}$, $N_{1}$, что для любых $k_{1}, j, 1 \leqslant j \leqslant D_{1}+N_{1} k_{1}=s_{1}$, и любых $(\xi, \eta), \eta \neq 0 \ldots 0$, $|(\xi, \eta)|=s_{1}$, на $\left(D_{1}, s_{1}, j\right)$-эксперименте вида $(1,0),(0,1),(0,1) \ldots(0,1)$ не сохраняется прямая зависимость с коэффициентами $(\xi, \eta)$. Взяв в качестве $g$ функцию от одного переменного, равную нулю, получим, что найдутся $D_{2}, N_{2}$ такие, что для всех $k_{2}, j, 1 \leqslant j \leqslant D_{2}+N_{2} k_{2}=s_{2}$, и любого $\xi \in E_{2}^{s_{2}}$ на $\left(D_{2}, s_{2}, j\right)$-эксперименте вида $(1,0)(0,0) \ldots(0,0)$ не сохраняется прямая зависимость с коэффициентами $(\xi, 0 \ldots 0)$. Проведя аналогичные рассуждения для двойственной зависимости получим, что для любого $N$ найдутся $k_{0}, D_{0}$ такие, что для всех $k$ и всех $D \geqslant D_{0}$

$$
\Omega_{1}\left(N, D, k_{0} k\right)=\Omega_{2}\left(N, D, k_{0} k\right)=\Omega_{3}\left(N, D, k_{0} k\right)=\Omega_{4}\left(N, D, k_{0} k\right)=\varnothing .
$$

Пусть $\Omega_{6}(N, D, k) \neq \varnothing$ для $N$ и для любых $D, k$. Без ограничения общности предположим, что $\left(G_{1}, G_{2}, G_{3}\right) \in \Omega_{6}(N, D, k)$ для бесконечного числа $D, k$, и в каждом из этих случаев сохраняется тождественная зависимость в момент $i(D, k)$, $D \leqslant i(D, k) \leqslant j(D, k)$, на $(D, D+k N, j(D, k))$-экспериментах. Это означает, что для некоторых $\beta=0 \ldots 0, \xi_{1}=0 \ldots 0, \xi_{2}=0 \ldots 010 \ldots 0, \xi_{3}=0 \ldots 0,|\beta|,\left|\xi_{l}\right| \leqslant|G Z|$, $l=1,2,3,|\beta|=r,\left|\xi_{2} \xi_{3}\right|=R$, справедливы равенства

$$
\begin{aligned}
& \phi_{Z}\left(G_{0}, \xi_{1}\right)=\phi_{Z}\left(G_{0}, \xi_{1} \beta\right)=G_{1}, \\
& \phi_{Z}\left(G_{0}, \xi_{2}\right)=\phi_{Z}\left(G_{0}, \xi_{2} \beta\right)=G_{2}, \\
& \phi_{Z}\left(G_{0}, \xi_{3}\right)=\phi_{Z}\left(G_{0}, \xi_{3} \beta\right)=G_{3} .
\end{aligned}
$$

Пусть $N_{0}=2 k_{0} N r R$ и $a$-функция $g$, реализующая счетчик $B_{D, N_{0}}$, выразима через $H$ и $f$; тогда найдутся $L_{0}, D_{0}$ такие, что для $s=D_{0}+L_{0} N$ и всех $j=D_{0}, \ldots, D_{0}+L_{0} N$ найдется $(D, s, j)$-эксперимент, на котором не сохраняется тождественная зависимость в $i$-й момент для $|i-j|<L_{0} N$. Но тогда и для коэффициентов $\xi_{1}, \xi_{2}, \hat{\xi_{3}}=0 \ldots 0,\left|\xi_{2} \hat{\xi_{3}}\right|=L_{0} N_{0}$, сохраняется тождественная зависимость на всех $\left(D_{0}, D_{0}+L_{0} N, j^{\prime}\right)$-экспериментах в $i^{\prime}$-й момент и $\left|i^{\prime}-j^{\prime}\right|<N_{0}$, что противоречит предположению о выразимости $a$-функции $g$. Для $\xi_{3}=0 \ldots 010 \ldots 0$ получается сохранение тождественной зависимости в момент $i^{\prime}$ и $\left|j^{\prime}+L_{0} N-i^{\prime}\right|<N$, что также противоречит предположению о выразимости $a$-функции $g$. Следовательно, $\Omega_{6}\left(N, D_{0}, L_{0}\right)=\varnothing$. Случаи $\Omega_{5}, \Omega_{7}, \Omega_{8}$ рассматриваются аналогично. Необходимость доказана.

Докажем достаточность. Зафиксируем $N$ и построим из $a$-функций $f, H a$ функцию счетчик $B_{D_{0}, N}$ для некоторого $D_{0}$. По условию леммы найдутся такие $D$ и $k$, что при $s=D+k N$ для любого $1 \leqslant j \leqslant s$ и любых $(\xi, \eta) \in\left(E_{2}^{2}\right)^{s}$ найдется $(D, s, j)$ эксперимент с $f$, на котором не выполнена прямая зависимость с коэффициентами $(\xi, \eta)$, найдется $(D, s, j)$-эксперимент с $f$, на котором не выполнена двойственная зависимость с коэффициентами $(\xi, \eta)$, а также для любых $i, j, 1 \leqslant i, j \leqslant s$, найдется указанный эксперимент, не сохраняющий инверсную зависимость в $i$-й момент и (при $i \neq j$ ) тождественную зависимость.

Зафиксируем $D, s, j$; пусть $R_{j}$ - число разных $(D, s, j)$-экспериментов, $f_{j}$ - параллельное соединение $R>R_{j}$ копий $f$. Пусть $(D, s, j)$-эксперимент (2) $a$-функции $f_{j}$ - это декартово произведение всех разных $(D, s, j)$-экспериментов $a$-функции $f$. Можно считать (за счет добавки фиктивных входов к $f_{j}$ ), что $m+n$ четно и каждое из $(a(i), b(i))$ содержит ровно $(m+n) / 2$ единиц, и среди наборов $(a(i), b(i))$ нет попарно двойственных. Зафиксируем в $(2)(a(1), b(1)), \ldots(a(s), b(s))$, 
тогда для переменного $a$ справедливо следующее утверждение. Для $(\xi, \eta)$ найдутся $a_{(\xi, \eta)}, \tilde{a}_{(\xi, \eta)}$ такие, что при $a=a_{(\xi, \eta)}(2)$ не сохраняет прямую зависимость с коэффициентами $(\xi, \eta)$, при $a=\tilde{a}_{(\xi, \eta)}(2)$ не сохраняет двойственную зависимость с коэффициентами $(\xi, \eta)$, кроме того, если $a=a(i)$, то $b \neq b(i)$ при $i \neq j$; если $a=0$ то $b \neq 0$; если $a=1$ то $b \neq 1$; если $a=\bar{a}(i)$, то $b \neq \bar{b}(i)$.

Пусть $F \subset E_{2}^{m+n}, F=\{a(i), b(i) \mid i=1, \ldots, s\}, \tilde{F}-$ множество наборов длины $m+n$, содержащих меньше, чем $(m+n) / 2$ единиц, $\hat{F}-$ множество наборов, содержащее больше $(m+n) / 2$ единиц, и множество $F^{\prime}$ содержит ровно $(m+n) / 2$ единиц. Пусть

$$
M=\left\{(a, b) \mid a \in E_{2}^{n}, b \in E_{2}^{m}, b=\psi(q(j), a)\right\} .
$$

Определим $h_{1}: E_{2}^{m+n} \rightarrow E_{2}^{n}, h_{1} \in\{H\}$, полагая

$$
h_{1}(x, y)= \begin{cases}x, & (x, y) \in F \\ a(j), & (x, y) \in\left(F^{\prime} \backslash F\right) \cap M, \\ 1, & (x, y) \in \hat{F} \cap M, \\ 0, & (x, y) \in \tilde{F} \cap M .\end{cases}
$$

Пусть $g_{0}(X)=h_{1}\left(X, f_{j}(X)\right)$. Для $а$-функции

$$
g_{1}(X)=\left(g_{0}(X), f_{j}\left(g_{0}(X)\right)\right.
$$

рассмотрим $(D, s, j)$-эксперимент при переменном $a \in E_{2}^{n}$

$$
(a,(c, d)),(a(1),(a(1), b(1)), \ldots,(a(s),(a(s), b(s))) .
$$

Тогда $(c, d) \in\left\{(a(j), b(j)),\left(1, d_{1}\right),\left(0, d_{0}\right)\right\}$, где $d_{1}=\psi(q(j), 1), d_{0}=\psi(q(j), 0)$. Пусть

$$
\begin{aligned}
& I_{1}=\left\{i \mid(a(i), b(i)) \vee\left(1, d_{1}\right)=1\right\}, \\
& J_{1}=\left\{i \mid(a(i), b(i)) \leqslant\left(1, d_{1}\right)\right\} .
\end{aligned}
$$

Если $I_{1}$ и $J_{1}$ одновременно не пусты, то определим $h_{2}: E_{2}^{m+n} \rightarrow E_{2}^{n}, h_{2} \in[\{H\}]$, полагая

$$
h_{2}(x, y)= \begin{cases}x, & (x, y) \in F^{\prime}, \\ a_{\left(\xi_{1}, \eta_{1}\right)}, & (x, y)=\left(1, d_{1}\right), \\ 0, & (x, y)=\left(0, d_{0}\right),\end{cases}
$$

где $\xi_{1}(i)=1$ при $i \in I_{1}, \xi_{1}(i)=0$ при $i \notin I_{1}, \eta_{1}(i)=1$ при $i \in J_{1}, \eta_{1}(i)=0$ при $i \notin J_{1}$. Для $a$-функции

$$
g_{2}(X)=\left(g_{1}(X), h_{2}\left(g_{1}(X)\right), f_{j}\left(h_{2}\left(g_{1}(X)\right)\right)\right)
$$

рассмотрим $(D, s, j)$-эксперимент при переменном $a$

$$
(a, c, d, e, f)(a(1), a(1), b(1), a(1), b(1)), \ldots,(a(s), a(s), b(s), a(s), b(s)) \text {; }
$$

тогда $(c, d, e, f) \in\left\{(a(j), b(j), a(j), b(j)),\left(1, d_{1}, a_{\left(\xi_{1}, \eta_{1}\right)}, d_{2}\right),\left(0, d_{0}, 0, d_{0}\right)\right\}$, где $d_{2}=$ $\psi\left(q(j), a_{\left(\xi_{1}, \eta_{1}\right)}\right)$. Пусть

$$
\begin{aligned}
& I_{2}=\left\{i \mid(a(i), b(i), a(i), b(i)) \vee\left(1, d_{1}, a_{\left(\xi_{1}, \eta_{1}\right)}, d_{2}\right)=1\right\} \\
& J_{2}=\left\{i \mid(a(i), b(i), a(i), b(i)) \leqslant\left(1, d_{1}, a_{\left(\xi_{1}, \eta_{1}\right)}, d_{2}\right)\right\}
\end{aligned}
$$


Тогда либо $J_{2} \subset J_{1}$, либо $I_{2} \subset I_{1}$. Если $I_{2}$ и $J_{2}$ одновременно не пусты, то определим $h_{3}: E_{2}^{m+n} \times E_{2}^{m+n} \rightarrow E_{2}^{n}$, полагая

$$
h_{3}(x, y, u, v)= \begin{cases}x, & (x, y, x, y) \in F \times F \\ a_{\left(\xi_{2}, \eta_{2}\right)}, & (x, y, u, v)=\left(1, d_{1}, a_{\left(\xi_{1}, \eta_{1}\right)}, d_{2}\right) \\ 0, & (x, y, u, v)=\left(0, d_{0}, 0, d_{0}\right),\end{cases}
$$

где $\xi_{2}(i)=1$ при $i \in I_{2}, \xi_{2}(i)=0$ при $i \notin I_{2}, \eta_{2}(i)=1$ при $i \in J_{2}, \eta_{2}(i)=0$ при $i \notin J_{2}$, и рассмотрим

$$
g_{3}(X)=\left(g_{2}(X), h_{3}\left(g_{2}(X)\right), f_{j}\left(h_{3}\left(g_{2}(X)\right)\right)\right)
$$

и множества

$$
\begin{aligned}
& I_{3}=\left\{i \mid(a(i), b(i), a(i), b(i), a(i), b(i)) \vee\left(1, d_{1}, a_{\left(\xi_{1}, \eta_{1}\right)}, d_{2}, a_{\left(\xi_{2}, \eta_{2}\right)}, d_{3}\right)=1\right\} \\
& J_{3}=\left\{i \mid(a(i), b(i), a(i), b(i), a(i), b(i)) \leqslant\left(1, d_{1}, a_{\left(\xi_{1}, \eta_{1}\right)}, d_{2}, a_{\left(\xi_{2}, \eta_{2}\right)}, d_{3}\right)\right\}
\end{aligned}
$$

для которых либо $I_{3} \subset I_{2}$, либо $J_{3} \subset J_{3}$. Этот процесс закончится, когда $I_{l+1}=\varnothing, J_{l+1}=\varnothing$ для некоторого $l$, и функция $h_{l+1}:\left(E_{2}^{m+n}\right)^{l} \rightarrow E_{2}^{n}$, $h_{l+1} \in[\{H\}]$, удовлетворяет условиям $h_{l+1}(x, y, x, y, \ldots, x, y)=x$ при $(x, y) \in F$, $h_{l+1}\left(1, d_{1}, a_{\left(\xi_{1}, \eta_{1}\right)}, d_{2}, \ldots a_{\left(\xi_{l}, \eta_{l}\right)}, d_{l}\right)=a(j), h_{l+1}\left(0, d_{0}, \ldots, 0, d_{0}\right)=0$.

$A$-функция $g(X)=g_{l+1}(X)$ имеет $(D, s, j)$-путь $(2)$ такой, что

$$
\left\{\psi(q(j), a) \mid a \in E_{2}^{n}\right\}=\{0, a(j)\} .
$$

Пусть

$$
\begin{aligned}
& \tilde{J}_{1}=\left\{i \mid(a(i), b(i)) \geqslant\left(0, d_{0}\right)\right\}, \\
& \tilde{I}_{1}=\left\{i \mid\left((a(i), b(i)) \wedge\left(0, d_{0}\right)\right)=0\right\} .
\end{aligned}
$$

Аналогично предыдущему, построим цепочку множеств

$$
\tilde{I}_{1} \supset \tilde{I}_{2} \supset \ldots \supset \tilde{I}_{k}=\varnothing, \quad \tilde{J}_{1} \supset \tilde{J}_{2} \supset \ldots \supset \tilde{J}_{k}=\varnothing,
$$

где вместо $а$-функции $f$ будет использоваться $a$-функция $g$. Полученная таким способом из $g, H$-функция $C_{j}$ имеет $(D, s, j)$-эксперимент (2) такой, что $\psi(q(j), a)=a(j)$ при всех $a \in E_{2}^{n} . A$-функция $C=f\left(C_{1}\left(C_{2}\left(\ldots C_{s}(x) \ldots\right)\right)\right)$ имеет $(D, s, j)$-эксперимент (2), в каждом состоянии $l, l=1,2, \ldots s$, которого реализуется константа $(a(l), b(l))$. Применив к этой $a$-функции операцию обратной связи, получим константную $a$-функцию $\Omega \quad$ с выходным словом $(a(1), b(1)) \ldots(a(s), b(s))(a(D), b(D)) \ldots(a(s), b(s)) \ldots$ Найдется $\delta \in[\{H\}]$ такая, что $\delta(a(j), b(j))=0 \ldots 010 \ldots 0$ для всех $j$, где 1 стоит на $j$-том месте, следовательно, $B_{D, N} \in[\{\delta, f\}]$. Найдутся также такие $\delta_{0}, \delta_{1} \in[\{H\}]$, что $\delta_{0}(a(j), b(j))=$ $0, \delta_{1}(a(j), b(j))=1, j=1,2, \ldots, s$. Значит, из $a$-функции $f$ можно получить все константные $a$-функции, в том числе константы 0 и 1 .

Система $\{0,1, H\}$ порождает все монотонные функции, в том числе

$$
\begin{aligned}
\delta_{0}\left(x_{1}, \ldots, x_{D+N}, y_{1}, \ldots, y_{D+N}\right)=x_{1} y_{1} \vee & \ldots \vee x_{D+N} y_{D+N} \\
& x_{1}, \ldots x_{D+N} \in E_{2}^{k}, \quad y_{1}, \ldots y_{D+N} \in E_{2} .
\end{aligned}
$$


Для любого $k$ можно получить $a$-функцию $(D, N)$-селектор

$$
C_{D, N}\left(X_{1}, \ldots X_{D+N}\right)=\delta_{0}\left(X_{1}, \ldots X_{D+N}, B_{D, N}\right) .
$$

Зафиксируем $j, 1 \leqslant j \leqslant D+N$. Для $(\xi, \eta)$, где $\xi=0 \ldots 0, \eta=0 \ldots 010 \ldots 0$, где 1 стоит на $j$-м месте, несохранение прямой зависимости означает, что для некоторого $a$ в (3) $\bar{a} a(j)=0, \bar{b} b(j) \neq 0$, т.е. $a \geqslant a(j), b \ngtr / b(j)$. Пусть $\delta_{3, j}, \delta_{4, j}-$ монотонные вектор-функции $\delta_{3, j}: E_{2} \rightarrow E_{2}^{n}, \delta_{4, j}: E_{2}^{m} \rightarrow E_{2}$, такие, что $\delta_{3, j}(0)=$ $a(j), \delta_{3, j}(1)=a, \delta_{4, j}(b)=0, \delta_{4, j}(b(j))=1$. Пусть $f_{j}^{\prime}: E_{2}^{*} \rightarrow\left(E_{2}^{n}\right)^{*}, f_{j}^{\prime \prime}:\left(E_{2}^{m}\right)^{*} \rightarrow E_{2}^{*}$, $v_{j}: E_{2}^{*} \rightarrow E_{2}^{*}$ таковы, что

$$
\begin{gathered}
f_{j}^{\prime}(X)=C_{D, N}\left(a(1), \ldots a(j-1), \delta_{3, j}(X), a(j+1), \ldots, a(D+N)\right), \\
f_{j}^{\prime \prime}=C_{D, N}\left(0, \ldots, 0, \delta_{4, j}, 0, \ldots, 0\right), \quad v_{j}=f_{j}^{\prime \prime}\left(f\left(f_{j}^{\prime}\right)\right) .
\end{gathered}
$$

Тогда $a$-функция $v_{j}$ имеет $(D, s, j)$-эксперимент вида

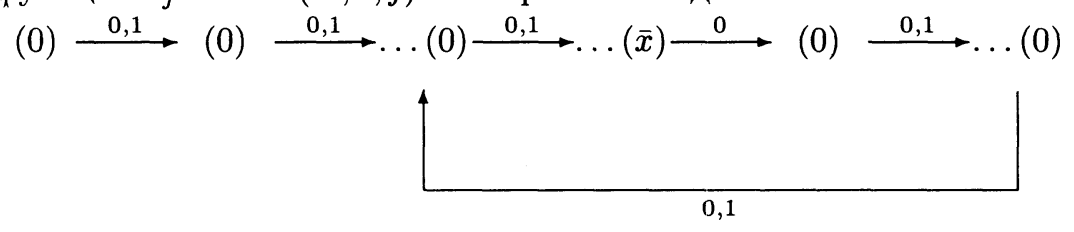

Выбрав $\delta_{3, j}^{\prime}(1)=a(j), \delta_{3, j}^{\prime}(0)=a, \delta_{4, j}^{\prime}(b)=1, \delta_{4, j}^{\prime}(b(j))=0$, получим $w_{j}$, имеющую $(D, s, j)$-эксперимент вида

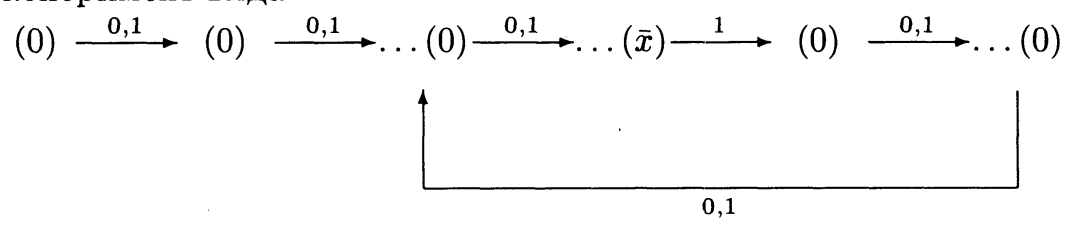

Пусть $a$-функция $u_{j}$ выразима через $C_{D, N}, B_{D, N}, H, 0,1$ и имеет диаграмму

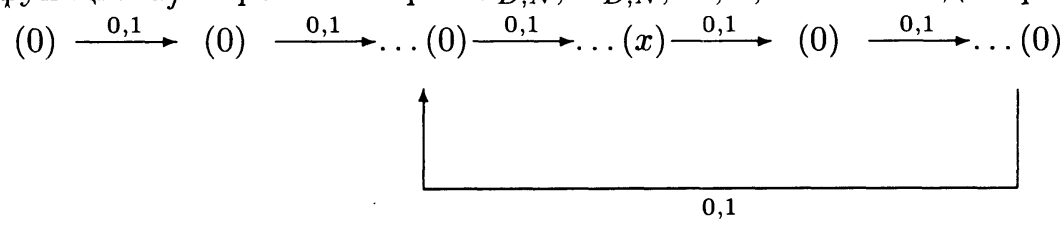

Тогда $\Delta_{j}(X, Y)=w_{j}(X \vee Y) \vee\left(v_{j}(X \wedge Y) \wedge u_{j}(Y)\right)$ после применения обратной связи ко входу $Y$ даст $\Gamma_{j}(X)$ с диаграммой

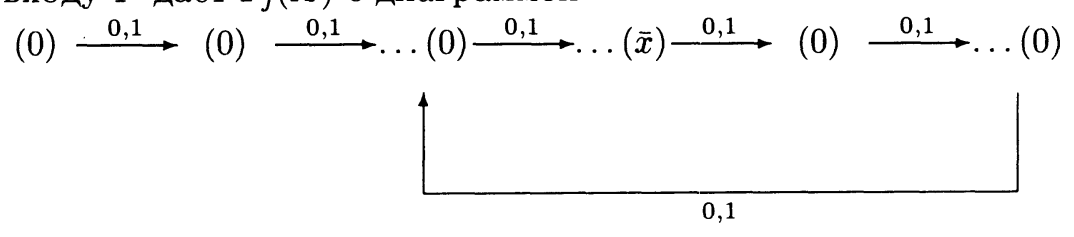

Положим

$$
\Gamma(X)=\bigvee_{1}^{s} \Gamma_{j}(X)=\bar{X} .
$$

Согласно [3], система $\{f, H\}$ полна. Лемма доказана. 
Лемма 2 доказывается аналогично.

\section{Список литературы}

1. Кудрявцев В. Б. О мощности множеств предполных множеств некоторых функциональных систем, связанных с автоматами. Пробл. киберн. (1965) 13, 45-74.

2. Кратко М. И. Алгоритмическая неразрешимость проблемы распознавания полноты для конечных автоматов. Докл. АН ССCР (1964) 155, №1, 35-37.

3. Бабин Д. Н. Разрешимый случай задачи о полноте автоматных функций. Дискретная математика (1992) 4, №4, 41-45.

4. Буевич В. А. Об алгоритмической неразрешимости распознавания $A$-полноты. $M a$ тем. заметки (1972) 12, №6, 687-697.

5. Буевич В. А. Условия А-полноты для конечных автоматов. Изд-во МГУ, Москва, 1986.

6. Кудрявцев В. Б., Гаврилов Г. П., Яблонский С. В. Функции алгебры логики и классы Поста. Наука, Москва, 1966.

7. Кудрявцев В. Б., Алешин С. В., Подколзин А. С. Введение в теорию автоматов. Наука, Москва, 1985.

8. Мальцев А. И. Итеративные алгебры Поста. Изд-во ИМСО АН СССР, Новосибирск, 1976.

9. Мальцев А. И. Алгоритмы и рехурсивные фунжции. Наука, Москва, 1986.

10. Бабин Д. Н. Неразрешимость проблемы полноты и $A$-полноты некоторых систем а-функций. Дискретная математика (1995) 7, №1, 52-65.

Статья поступила 04.01.95. 